\title{
Generation and selection of immunized Fab phage display library against human B cell lymphoma
}

\author{
Yongmei Shen ${ }^{1,2}$, Xiaochun Yang ${ }^{1}$, Ningzheng Dong ${ }^{3}$, Xiaofang Xie ${ }^{1,2}, \mathrm{Xia} \mathrm{Bai}^{3}$, Yizhen $\mathrm{Shi}^{1,2}$ \\ ${ }^{1}$ Department of Radioimmunoassay, ${ }^{2}$ Department of Clinical Laboratory, Second Affiliated Hospital of Soochow University, Suzhou \\ 215004, China; ${ }^{3}$ Leukemia Research Unit, Jiangsu Institution of Hematology, First Affiliated Hospital of Soochow University, Suzhou \\ 215006, China
}

The approval of using monoclonal antibodies as a targeted therapy in the management of patients with B cell lymphoma has led to new treatment options for this group of patients. Production of monoclonal antibodies by the traditional hybridoma technology is costly, and the resulting murine antibodies often have the disadvantage of triggering human anti-mouse antibody (HAMA) response. Therefore recombinant Fab antibodies generated by the phage display technology can be a suitable alternative in managing B cell lymphoma. In this study, we extracted total RNA from spleen cells of BALB/c mice immunized with human B lymphoma cells, and used RT-PCR to amplify cDNAs coding for the $\kappa$ light chains and $\mathrm{Fd}$ fragments of heavy chains. After appropriate restriction digests, these cDNA fragments were successively inserted into the phagemid vector pComb3H-SS to construct an immunized Fab phage display library. The diversity of the constructed library was approximately $1.94 \times 10^{7}$. Following five rounds of biopanning, soluble Fab antibodies were produced from positive clones identified by ELISA. From eight positive clones, FabC06, FabC21, FabC43 and FabC59 were selected for sequence analysis. At the level of amino acid sequences, the variable heavy domains $\left(\mathrm{V}_{\mathrm{H}}\right)$ and variable light domains $\left(\mathrm{V}_{\mathrm{L}}\right)$ were found to share $88-92 \%$ and $89-94 \%$ homology with sequences coded by the corresponding murine germline genes respectively. Furthermore, reactivity with membrane proteins of the B cell lymphoma was demonstrated by immunohistochemistry and western blotting. These immunized Fab antibodies may provide a valuable tool for further study of B cell lymphoma and could also contribute to the improvement of disease therapy.

Keywords: B cell lymphoma, Fab, phage display library, pComb3H-SS vector

Cell Research (2007) 17:650-660. doi: 10.1038/cr.2007.57; publication online 10 July 2007

\section{Introduction}

The effects of various available treatments against malignant lymphoma, including conventional chemotherapy, radiotherapy, bone marrow transplantation and peripheral blood stem cell transplantation, are usually not completely satisfactory. With the discovery of hybridoma technology, monoclonal antibody (mAb)-based targeting anticancer therapy has provided an alternative approach to treating this malignant disease [1]. However, early clinical trials using murine mAbs suffered from human anti-mouse antibody

Correspondence: Yongmei Shen

Tel: + 86-512-67783445; Fax: +86-512-68284303

E-mail: yongmei_shen@yahoo.com

Received 20 August 2006; revised 1 March 2007; accepted 23 March 2007; published online 10 July 2007 response (HAMA), especially when multiple infusions were required to obtain therapeutic efficacy $[2,3]$. These host responses considerably altered the pharmacokinetic profile of the antibody, which led to rapid clearance of $\mathrm{mAb}$ and prevented repeated dosing [4]. Although many patients with B cell lymphoma have compromised immune systems, HAMA reactions are still problematic. Therefore, techniques to ameliorate the HAMA response by developing less immunogenic mAbs are required, including chimerization, humanization and development of human antibodies from phage display libraries [5-7]. Several examples of clinical antibody humanization have been described. The human anti-murine antibody response could be minimized by replacing murine sequences with homologous sequences derived from the human framework. Rituximab used in the therapy of B cell lymphoma is an 
example of a chimeric antibody and has produced some striking effects [8-10]. The construction and selection of phage antibody libraries represent an additional solution to the problem of HAMA response. In this approach, Fab fragments can be generated by a random combination of $\mathrm{Fd}$ chains (comprising the variable region $\left(\mathrm{V}_{\mathrm{H}}\right)$ and the first constant region $\left(\mathrm{C}_{\mathrm{H} 1}\right)$ of the heavy chain) and full-length light chains (comprising the variable region $\left(\mathrm{V}_{\mathrm{L}}\right)$ and the constant region $\left(\mathrm{C}_{\mathrm{L}}\right)$ of the light chain). Compared with the conventional hybridoma technology, this technique is relatively simple, and a considerable amount of antibodies could be obtained through a procedure called biopanning. Furthermore, in this approach, only Fab fragments of the antibodies are produced. It was suggested that antibody fragments had potential advantages over whole antibodies for some therapeutic purposes, since they are smaller and can thus better penetrate organs and tissues [11,12]. Therefore combinatorial phage display technology is now considered a very useful tool for the generation of both diagnostic and therapeutic mAbs $[13,14]$. Here we describe the construction of Fab antibodies against $\mathrm{B}$ cell lymphoma by the phage display technology. In addition, we have utilized a simple whole-cell-based biopanning procedure to isolate a panel of specific B cell lymphoma Fab antibodies.

\section{Materials and Methods}

\section{Vector, E. coli and helper phage}

The vector $\mathrm{pComb} 3 \mathrm{H}-\mathrm{SS}[15,16]$ (kindly provided by the Scripps Research Institute, La Jolla, CA, USA), a modified version of the original pComb3H phagemid vector [17], about 4915 base pairs, contains ampicillin resistance (Amp) gene, start sites of plasmid CO1E1 and F1 phage replication. The expression of inserting fragments is controlled by the same LacZ promoter on the upstream sequence of vector pComb3H-SS. E. coli XL1-Blue contains tetracycline resistance (Tet) gene on its gene type of Tn10. Helper phage (VCSM13) contains kanamycin resistance (Kana) gene with valency of $10^{12} \mathrm{pfu} / \mathrm{L}$. It is amplified in SOC culture medium and preserved at $4{ }^{\circ} \mathrm{C}$.

\section{Immunization of animals with B cell lymphoma strain}

Human B lymphoma cell strain Raji and Daudi were cultured in RPMI-1640 medium supplemented with 10\% FCS and antibiotics. $2 \times 10^{7} / \mathrm{ml}$ cells were used for the immunization of four BALB/c mice ( referred to as No.1, No.2, No.3 and No.4) by subcutaneous injection. The mice were given three boosts of the Raji cells and Daudi cells in a four week interval. Serum samples were titrated by indirect Enzyme-Linked Immunosorbent Assay (ELISA). Following the demonstration of sufficient antibody titer, the mice were given a final immunization with $8 \times 10^{7} / \mathrm{mL}$ cells. 3 days later, the mice were euthanized, exsanguinated, and the spleens were harvested.

\section{RNA isolation and RT-PCR amplification of heavy chain Fd and $\mathrm{\kappa}$ light chain}

Total RNA of the harvested mice spleen was extracted with Trizol
Reagent according to the manufacturer's procedure (Invitrogen, USA). The purity and concentration were determined by measuring the absorbance $(A)$ at $260 \mathrm{~nm}$ and $280 \mathrm{~nm}\left(\mathrm{~A}_{260} / \mathrm{A}_{280}\right)$. To generate first-strand cDNA, an oligo(dT) 18 was used as primer, and $30 \mu \mathrm{g}$ RNA was reverse-transcribed in the light of MMLV First Strand cDNA Synthesis Kit Protocols (Fermentas, USA). PCR amplification of heavy chain $\mathrm{Fd}$ and whole $\kappa$ light chain genes were performed with Taq polymerase and primers specific to murine $\mathrm{Fd}$ and Kappa light chain described in Table 1. PCR productions of amplified $\mathrm{V}_{\mathrm{H}}-\mathrm{C}_{\mathrm{H}}$ and $\mathrm{V}_{\mathrm{L}}-\mathrm{C}_{\mathrm{L}}$ from cDNA synthesis were gel purified according to QIAEXII Agarose Gel Extraction Protocols (Qiagen, USA), and then digested with excess restriction enzymes as detailed below.

\section{Library construction}

The $\kappa$ light chain products of PCR were digested with an excessive restriction enzymes $S a c \mathrm{I}$ and $X b a \mathrm{I}$ (Biolabs, USA), and about $4.0 \mu \mathrm{g}$ was ligated overnight with $40 \mu \mathrm{g}$ of $\mathrm{SacI} / \mathrm{XbaI}$ - linearlized pComb3H-SS vector (purified by agarose gel extraction) in a total volume of $200 \mu \mathrm{l}$ with 60 units of T4 DNA ligase (Promega, USA) at $16^{\circ} \mathrm{C}$. Following ligation, the recombinant DNA was precipitated, washed and dissolved in $15 \mu \mathrm{l}$ of distilled water. $1 \mu \mathrm{l}$ of recombinant DNA was transformed by electroporation [18] into $40 \mu 1$ of E. coli XL1-Blue every time. After transformation, $1 \mathrm{ml} \mathrm{SOC}$ medium was added and the culture was shaken for $1 \mathrm{~h}$ at $37^{\circ} \mathrm{C}$, and then $10 \mathrm{ml}$ of SB containing $20 \mu \mathrm{g} / \mathrm{ml}$ ampicillin and $10 \mu \mathrm{g} / \mathrm{ml}$ tetracycline was cultivated for an additional hour at $37^{\circ} \mathrm{C}$ on a shaker. At this point, culture aliquots were plated on LB agar/ampicillin to titer library size, which was calculated by counting the number of ampicillinresistant colonies. The insertion of target $\kappa$ fragments was detected by digestion with $S c a$ I and $X b a \mathrm{I}$. The culture was added to $100 \mathrm{ml}$ of SB containing $50 \mu \mathrm{g} / \mathrm{ml}$ ampicillin and $10 \mu \mathrm{g} / \mathrm{ml}$ tetracycline and was cultivated overnight. Phagemids containing the $\kappa$ light chains were prepared from this overnight culture and named pComb3H-S $+\kappa$. For cloning the heavy chain $\mathrm{Fd}$ fragments, the $\mathrm{Fd}$ products of

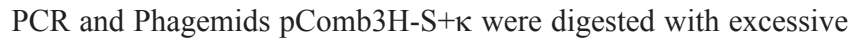
restriction enzymes $\mathrm{XboI}$ and SpeI. The ligation, transformation, $E$. coli amplification and determination of the library were performed as described above. The insertion of target $\mathrm{Fd}$ fragments was detected by digestion with XhoI and SpeI from the plasmids extracted from several random XL1-Blue monoclones. The Fab fragments' insertion was detected by digestion with SacI and SpeI. The Phagemids with $\mathrm{Fd}$ and $\kappa$ were named $\mathrm{pComb} 3 \mathrm{H}+\kappa+\mathrm{Fd}$. Approximately VCSM13 $\left(10^{12} \mathrm{pfu}\right)$ helper phage as described by de Alboran et al. [19] was added to XL1-Blue samples containing Fab gene libraries and incubated for $3 \mathrm{~h}$ at $37^{\circ} \mathrm{C}$ with shaking. $70 \mu \mathrm{g} / \mathrm{ml} \mathrm{Kanamycin} \mathrm{was}$ added to and the culture was shaken overnight at $37^{\circ} \mathrm{C}$. The cells were centrifuged at $4000 \mathrm{rpm}$ for $15 \mathrm{~min}$ at $4{ }^{\circ} \mathrm{C}$. The supernatant was mixed with $5 \mathrm{ml} 20 \%$ PEG 8000/2.5 M NaCl and incubated on ice for $30 \mathrm{~min}$, and then phages were precipitated by centrifugation at $8000 \mathrm{rpm}$ for $20 \mathrm{~min}$ at $4{ }^{\circ} \mathrm{C}$. The supernatant was discarded and the pellet drained. The phages were resuspended in $1.5 \mathrm{ml} \mathrm{PBS} / 1 \%$ BSA, vortexed and centrifuged at $13000 \mathrm{rpm}$ for 5 min to pellet debris. The supernatant was stored at $4{ }^{\circ} \mathrm{C}$ or used directly for the next round of biopanning.

\section{Biopanning of the primer Fab phage antibody library}

6-well plate (Nunclon ${ }^{\mathrm{TM}}$, Denmark) was coated by $5 \times 10^{6}$ cells with equal Raji and Daudi cells, and then blocked overnight with $2 \% \mathrm{BSA} / \mathrm{PBS}$ at $4{ }^{\circ} \mathrm{C} .1 \mathrm{ml}$ of phage library was added to each well 
Table I Primers for PCR amplification of murine Fd and Kappa light chain genes

\begin{tabular}{|c|c|}
\hline Primers & Sequences \\
\hline \multicolumn{2}{|l|}{ Heavy Chain Fd 3' primers } \\
\hline $\operatorname{IgG} 1$ & 5'- AGG CTT ACT AGT ACA ATC CCT GGG CAC AAT-3' \\
\hline $\operatorname{IgG} 3$ & 5'-GGG GGT ACT AGT CTT GGG TAT TCT AGG CTC-3' \\
\hline \multicolumn{2}{|l|}{ Heavy Chain $\mathrm{Fd} 5^{\prime}$ primers } \\
\hline He 3 & 5'-AGG TCC AGC TTE TCG AGT CTG G-3' \\
\hline Hc 4 & 5'-AGG TCC AGC TTC TCG AGT CAG G-3' \\
\hline Hc 5 & 5'-AGG TCC AAC TGC TCG AGT CTG G-3' \\
\hline Hc 6 & 5'-AGG TCC AAC TGC TCG AGT CAG G-3' \\
\hline$\kappa$ light chain $3^{\prime}$ primer & 5'-GCG CCG TCT AGA ATT AAC ACT CAT TCC TGT TGA A-3' \\
\hline \multicolumn{2}{|l|}{$\kappa$ light chain $5^{\prime}$ primers } \\
\hline Lc 1 & 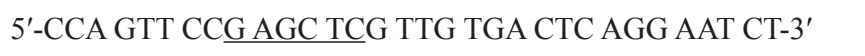 \\
\hline Lc 2 & 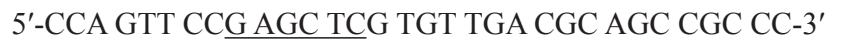 \\
\hline Lc 3 & 5'-CCA GTT CCG AGC TCG TGC TCA CCC AGT CTC CA-3' \\
\hline $\operatorname{Lc} 4$ & 5'-CCA GTT CCG AGC TCC AGA TGA CCC AGT CTC CA-3' \\
\hline Lc 5 & 5'-CCA GAT GTG AGC TCG TGA TGA CCC AGA CTC CA-3' \\
\hline Lc 6 & 5'-CCA GAT GTG AGC TCG TCA TGA CCC AGT CTC CA-3' \\
\hline Lc 7 & 5'-CCA GTT CCG AGC TCG TGA TGA CAC AGT CTC CA-3' \\
\hline
\end{tabular}

Footnote: Restriction enzymes recognition sites are underlined, with $\underline{\mathrm{A} \mid \mathrm{CTAGT}}$ for SpeI, $\underline{\mathrm{C} \mid \mathrm{TCGAG}}$ for XhoI, T|CTAGA for XbaI and $\underline{\mathrm{GAGCT} \mid \mathrm{C}}$ for $\mathrm{SacI}$.

and incubated at $37^{\circ} \mathrm{C}$ for $2 \mathrm{~h}$. Unbound phages were washed 1,5 , 10, 10, 10 times with TBS/ $0.5 \%$ Tween 20 (TBST) for rounds 1,2 , 3,4 and 5 respectively. Bound phages were eluted by adding elution buffer $(0.1 \mathrm{M}$ HCL, adjusted to $\mathrm{pH} 2.2$ with solid glycine and containing $0.1 \% \mathrm{BSA}$ ) and the eluted solution was neutralized with $2 \mathrm{M}$ Tris buffer, $\mathrm{pH}$ 8.0. Amplification procedures with VCSM13 helper phage were followed as described above, while input and output phages were titrated on LB-ampicillin plates. Phage binding, elution, and amplification steps were performed 5 rounds over consecutive days.

\section{Soluble expression of the Fab}

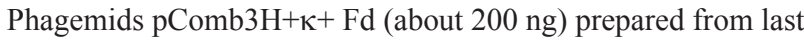
biopanning rounds were extracted and digested with the restriction enzymes NheI and SpeI to remove gene III fragments. The self-ligated phagemids without gene III fragments were transformed into XL1-Blue cells. Transformants were grown on LB plates containing ampicillin. Clones were examined for the removal of gene III fragments by $\mathrm{Sac \textrm {I }} / \mathrm{Not} \mathrm{I}$ digestion, which yielded a $2.0 \mathrm{~kb}$ fragment. Clones were grown in $10 \mathrm{ml} \mathrm{SB}$ containing $20 \mathrm{mM} \mathrm{MgCL}_{2}$ and
$50 \mu \mathrm{g} / \mathrm{ml}$ ampicillin at $37{ }^{\circ} \mathrm{C}$ until the culture reached OD600 $=1.0$. At this point, Isopropyl- $\beta$-D-thiogalactopyranoside (IPTG) was added up to the final concentration of $1 \mathrm{mM}$ and then the culture was incubated overnight at $30^{\circ} \mathrm{C}$. The bacteria pelleted by centrifugation were resuspended in $1 \mathrm{ml}$ PBS and lysed by freezing and thawing six times. The debris were pelleted and centrifuged at $15000 \mathrm{rpm}$ at $4{ }^{\circ} \mathrm{C}$ for $15 \mathrm{~min}$. The supernatant containing soluble Fab was used directly for ELISA analysis or stored at $-20^{\circ} \mathrm{C}$.

\section{Analysis of soluble Fab reactivity by ELISA}

Each Fab clone solution $(100 \mu \mathrm{l})$ was incubated for $2 \mathrm{~h}$ at $37^{\circ} \mathrm{C}$ in triplicate wells of an ELISA plate that had been coated with Raji cells, Daudi cells, Patu8988 cells (pancreatic cancer cell strain) and A549 cells (lung cancer cell strain) respectively and blocked with $2 \%$ BSA as described above. After five times of washing with $\mathrm{PBS} / 0.05 \%$ Tween $20,50 \mu \mathrm{l}$ horseradish peroxidase (HRP)-conjugated goat anti-mouse Fab antibody (Sigma, USA) was added (1:2 000 in $\mathrm{PBS} / 2 \%(\mathrm{v} / \mathrm{v}) \mathrm{BSA})$ and incubated for $1.5 \mathrm{~h}$ at $37^{\circ} \mathrm{C}$. Following further ten times of washing, color was developed with $100 \mu 1$ 3,3',5, $5^{\prime}$ tetramethylbenzidine (TMB) substrate, and the reaction was stopped 


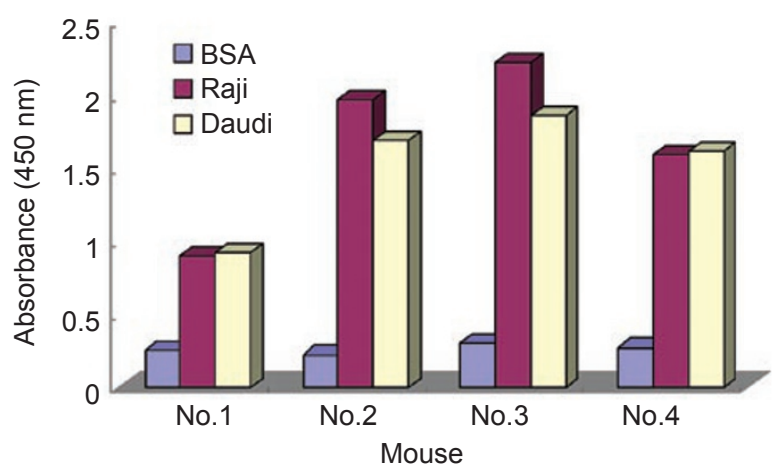

Figure 1 The titers of the antisera from BALB/c mice immunized with Raji cells and Daudi cells were analyzed by ELISA.

with $50 \mu$ of $2 \mathrm{M} \mathrm{H}_{2} \mathrm{SO}_{4}$. Clones were considered positive when the OD was more than 3 times the signal seen in wells not coated with Raji cells or Daudi cells. In each ELISA, a negative control without Fab was used to assess background signals.

\section{Sequence analysis}

The sequences of Fd and $\kappa$ light chain DNA fragments including clones FabC06, FabC21, FabC43 and FabC59 from the specific positive clones were determined by Shanghai Invitrogen Biotechnology Co.Ltd and their homology with murine Ig was analyzed according to GenBank. Primers used for sequencing the $\kappa$ light chain were 5'-GGAATTGTGAGCGGATAA-3' and 5'-CAGCGAGTAATAACAATCCAG- $3^{\prime}$. Primers used for sequencing the $\mathrm{Fd}$ fragment were 5'-AGCCGCTGGATTGTTATT-3' and 5'-GAACCGCCTCCCTCAGA-3'. The nucleotide sequences of $\mathrm{V}_{\mathrm{H}}$ and $\mathrm{V}_{\mathrm{L}}$ were translated into the sequences of amino acid, and their complementary determining regions (CDRs) and framework regions (FRs) were located according to Immunoglobin BLAST (available from http://www.ncbi.nlm.nih.gov/BLAST/).

\section{Western blot analysis}

To determine the molecular weight of the soluble Fab antibodies expressed by specific positive clones, the soluble Fab antibodies from FabC06 separated by $12 \%$ SDS-PAGE in reducing conditions
( with $\beta \mathrm{ME}$ ) as well as in non-reducing conditions (without $\beta \mathrm{ME}$ /DTT) were transferred to nitrocellulose membrane (Invitrogen, USA). After strips were blocked with 5\% fat-free milk powder for $1 \mathrm{~h}$ at room temperature, the Fab was detected by HRP conjugated goat anti-mouse Fab antibody (1:5 000). After being washed, the immunoreactive bands were visualized with ECL Reagent (Pufei Bio-tech, China) and exposed to x-ray film. An equal volume of pComb3H-SS plasmid transformed into XL1-Blue cell lysates served as the negative control for the Western blot experiment.

To investigate the specificity of soluble Fab antibodies toward the membrane antigens of B cell lymphoma, one million Raji cells and Daudi cells were lysed respectively in $100 \mu$ Cell Lysis Buffer (Beyotime Biotchnology, China). Protein products were separated by $12 \%$ SDS-PAGE under reducing conditions, transferred onto nitrocellulose membrane, probed with soluble Fab antibodies from FabC06 and FabC21, and followed by HRP conjucated goat ant-mouse Fab antibody (1:5 000). Bands were developed with ECL substrate and exposed to x-ray film.

\section{Analysis of Fab reactivity by immunohistochemistry}

In order to perform immunohistochemistry, $5 \mu \mathrm{m}$-thick paraffin-embedded B cell lymphoma tissue sections were cut, and subsequently dewaxed, re-hydrated, and then subjected to antigen retrieval heated in $10 \mathrm{mM}$ citrate buffer in a microwave for $15 \mathrm{~min}$. The sections were cooled, treated with $3 \% \mathrm{H}_{2} \mathrm{O}_{2}$, blocked with $10 \%$ goat serum, and then incubated overnight at $4{ }^{\circ} \mathrm{C}$ with the respective soluble Fab antibodies from FabC06, FabC21, FabC43 and FabC59. Negative controls were incubated with an equivalent volume of diluent solution alone. After 3 washes with PBS, the standard streptavidin-biotin-peroxidase complex technique by means of sequential 20 min incubation with biotinylated goat anti-mouse Fab (Sigma, USA) and peroxidase-labeled streptavidin (Invitrogen, USA) was performed. 3, 3'- diaminobenzidine (DAB) was used as a substrate chromogen solution for the development of peroxidase activity. Hematoxylin was used for nuclear counterstaining, and then the sections were mounted and cover-slipped.

\section{Results}

\section{Characterization of serum and selection of immunized} mice

Three days after the final booster injection with Raji and Daudi cells, sera from immunized mice were collected and analyzed by ELISA for the antibody response to tumor

Table 2 Selective enrichment of phages

\begin{tabular}{lcccc}
\hline Rounds of panning & Input Phages $(\mathrm{cfu})$ & Eluted Phages $(\mathrm{cfu})$ & Yield $(\%)$ & Washing times \\
\hline First & $4.00 \times 10^{11}$ & $3.00 \times 10^{5}$ & $7.50 \times 10^{-5}$ & $1.33 \times 10^{-5}$ \\
Second & $1.00 \times 10^{11}$ & $1.33 \times 10^{5}$ & $5.00 \times 10^{-4}$ & 5 \\
Third & $7.50 \times 10^{10}$ & $3.75 \times 10^{5}$ & $1.35 \times 10^{-3}$ & 10 \\
Fourth & $4.00 \times 10^{10}$ & $5.40 \times 10^{5}$ & $1.80 \times 10^{-2}$ & 10 \\
Fifth & $1.50 \times 10^{10}$ & $2.70 \times 10^{6}$ & 10 \\
\hline
\end{tabular}

Footnote: Yield $(\%)=($ No. of eluted phages $) \times 100 /($ No. of input phages $)$ 
cells, with BSA coated wells serving as the negative control. The results showed that three of the four mice had a strong antibody response to Raji cells and Daudi cells (Figure 1). The mice with the highest (No.3) and the second highest titers (No.2) were killed for Fab phage display library construction.

\section{RT-PCR amplification of heavy chain Fd and $\kappa$ light chain}

Total RNAs were isolated from spleen cells from the immunized mice (No.2 and No.3). The integrity of RNAs was checked by alkaline denaturing agarose gel electrophoresis. The $\mathrm{A}_{260} / \mathrm{A}_{280}$ was 2.09. cDNAs coding for the immunoglobulin $\mathrm{Fd}$ and $\kappa$ chains were amplified by a variety of primer combinations designed to amplify a majority of the known mouse antibody sequences, with suitable restriction sites for later cloning into the $\mathrm{pComb3H-SS}$ vector. The size of the amplified fragments of $\mathrm{Fd}$ and $\kappa$ was about $680 \mathrm{bp}$ (Figure 2).

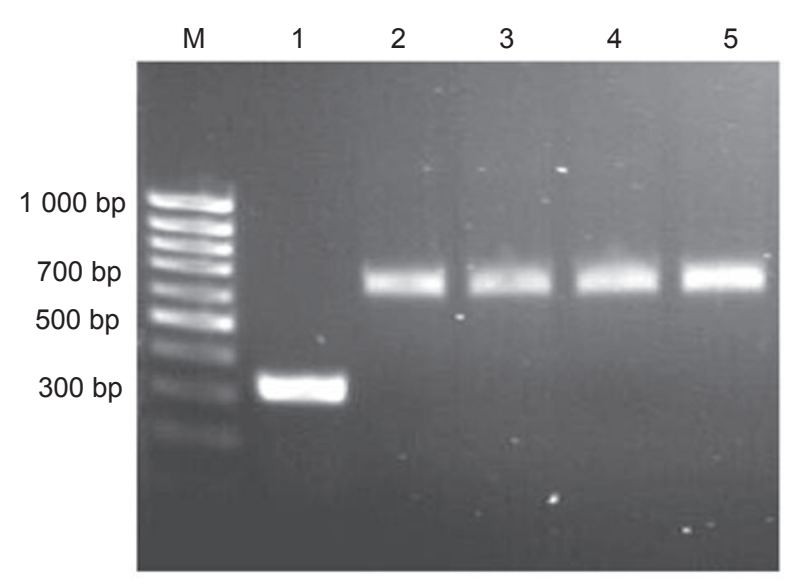

Figure 2 Amplification of cDNAs coding for the $\kappa$ light chains and the heavy chain Fd fragments by PCR. Lane M: 100 bp DNA marker; Lane 1: $\beta$-actin; Lane 2: Mixed $\kappa$ light chains; Lane 3: Mixed Fd fragments of heavy chains IgG1; Lane 4: Mixed Fd fragments of heavy chains IgG2a; Lane 5: Mixed Fd fragments of heavy chains IgG3.

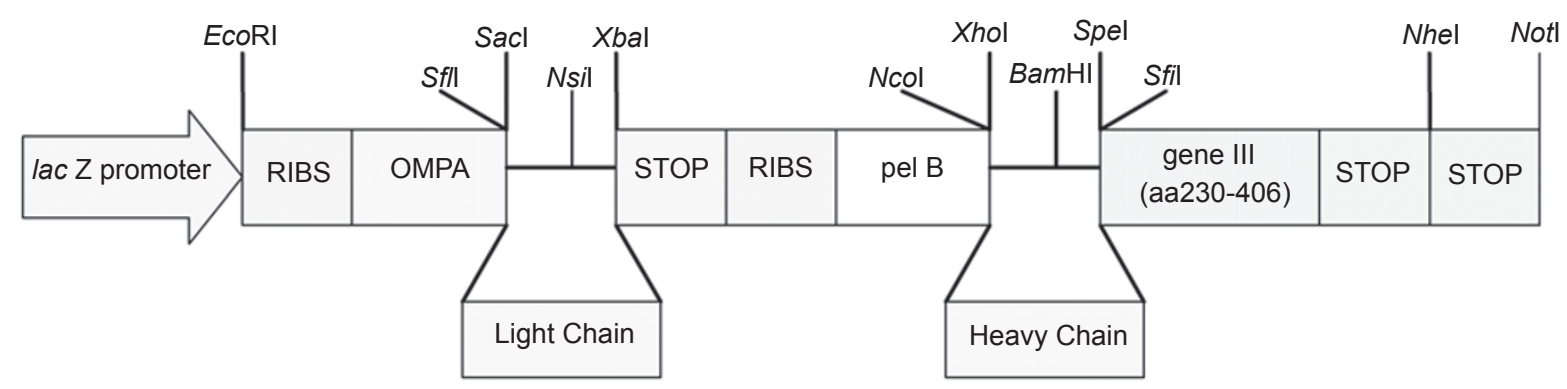

Figure 3 Structure of the phagemid vector pComb3H-SS. The vector is provided only in the pComb3H-SS form where the SS designation is used for the vector with stuffer fragments. The heavy chain stuffer is $300 \mathrm{bp}$ and the light chain stuffer is $1200 \mathrm{bp}$ of the sequence. The $\mathrm{SacI}$ and $\mathrm{XbaI}$ restriction sites are used for cloning of the light chain fragments whilst the Fd fragments are inserted at XhoI and SpeI sites. Cleavage of pComb3H by SpeI (A|CTAGT) and NheI (G|CTAGT) would produce compatible cohesive ends that can be re-ligated, resulting in the removal of the gene III product of VCSM13 producing soluble Fabs. The lacZ promoter drives the synthesis of the light chain and $\mathrm{Fd} /$ gene III transcript. The leader peptides ompA and pelB target these two polypeptides to the bacterial periplasm where the soluble light chain fragment and the membrane-bound Fd fragment associate via a disulfide bond.

Table 3 ELISA detection of reactivity between positive Fabs and various cells (mean $\pm \mathrm{SD}, \mathrm{A}_{450}$ value)

\begin{tabular}{cccccc}
\hline positive clones & Raji cells & Daudi cells & Patu 8988 & A549 & BSA \\
\hline FabC02 & $1.693 \pm 0.087$ & $0.497 \pm 0.038$ & $0.273 \pm 0.045$ & $0.294 \pm 0.052$ & $0.263 \pm 0.038$ \\
FabC06 & $1.886 \pm 0.059$ & $1.522 \pm 0.081$ & $0.249 \pm 0.028$ & $0.371 \pm 0.039$ & $0.271 \pm 0.031$ \\
FabC21 & $1.732 \pm 0.061$ & $1.542 \pm 0.092$ & $0.290 \pm 0.026$ & $0.342 \pm 0.045$ & $0.203 \pm 0.027$ \\
FabC36 & $1.519 \pm 0.033$ & $0.627 \pm 0.023$ & $0.298 \pm 0.031$ & $0.284 \pm 0.035$ & $0.297 \pm 0.032$ \\
FabC43 & $1.610 \pm 0.034$ & $1.763 \pm 0.044$ & $0.306 \pm 0.037$ & $0.345 \pm 0.020$ & $0.301 \pm 0.036$ \\
FabC48 & $1.536 \pm 0.054$ & $1.489 \pm 0.064$ & $0.364 \pm 0.031$ & $0.296 \pm 0.049$ & $0.276 \pm 0.038$ \\
FabC59 & $1.558 \pm 0.048$ & $1.792 \pm 0.036$ & $0.291 \pm 0.034$ & $0.327 \pm 0.027$ & $0.261 \pm 0.040$ \\
FabC65 & $1.597 \pm 0.037$ & $1.184 \pm 0.051$ & $0.323 \pm 0.030$ & $0.284 \pm 0.041$ & $0.317 \pm 0.022$ \\
\hline
\end{tabular}



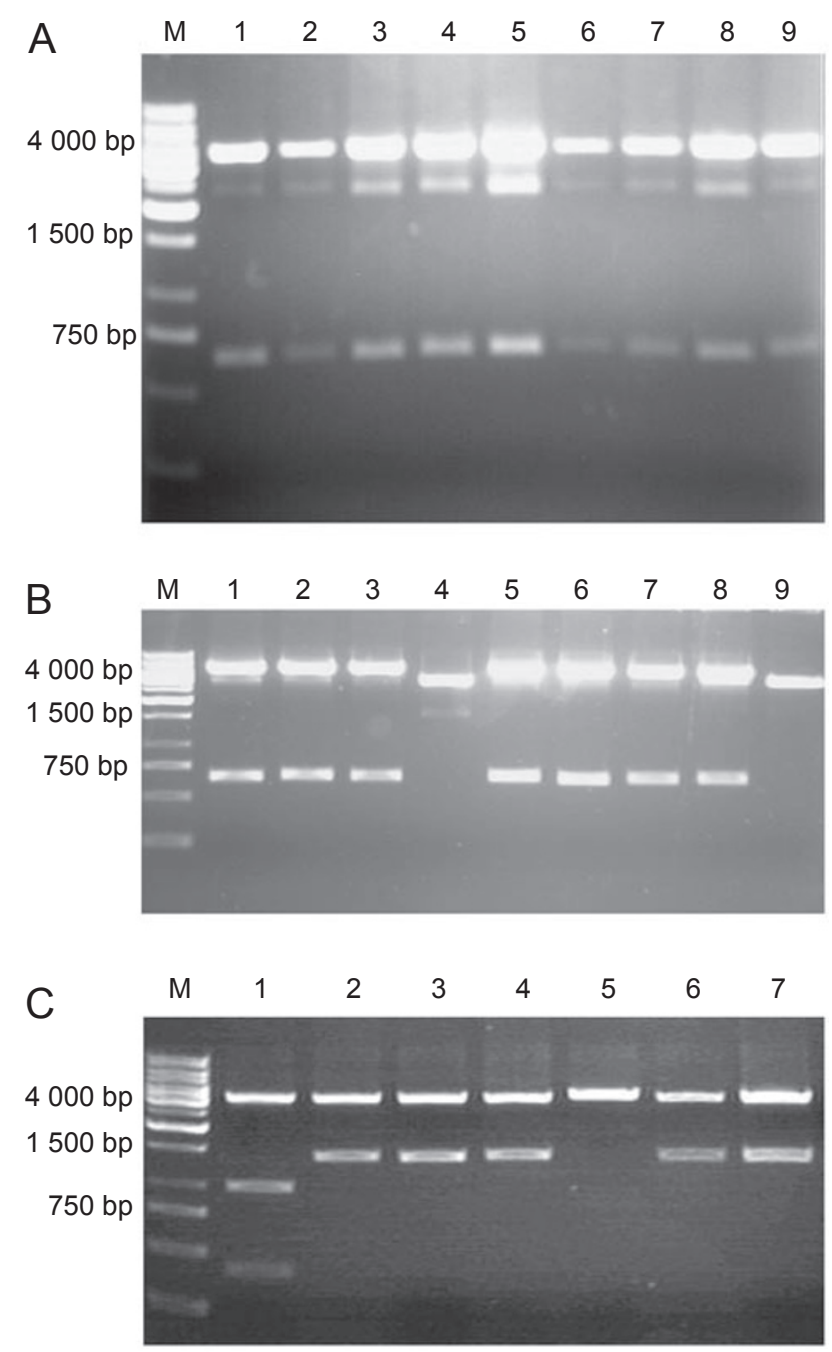

Figure 4 Fab library construction. (A) Identification of the $\kappa$ insert by $\mathrm{SacI} / \mathrm{Xba \textrm {I }}$ digestions. The $\kappa$ light chain $\mathrm{PCR}$ products were cloned between the $S a c I$ and $X b a \mathrm{I}$ sites of the pComb3H-SS vector and the $\kappa$ inserts were identified by $S a c \mathrm{I} / X b a \mathrm{I}$ digestions. Lane $\mathrm{M}: 1 \mathrm{~kb}$ DNA marker; Lane 1 to lane 9: nine randomly selected clones which contained the expected $\sim 680$ bp insert. (B) Fd insert identification by $X h o I / S p e I$ digestions. Phagemids containing the $\kappa$ light chain repertoires were named as pComb3H-S $+\kappa$. The heavy chain Fd PCR products digested with $\mathrm{XhoI} / \mathrm{SpeI}$ were cloned between $\mathrm{XhoI}$ and

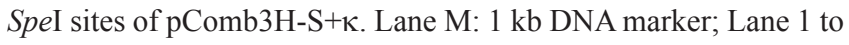
lane 9 show that 7 of the 9 randomly selected clones contained the $\sim 680$ bp Fd fragment. (C) Identification of Fab inserts by SacI/SpeI digestions. Lane M: $1 \mathrm{~kb}$ DNA marker; Lane 1 to lane 7 show that 5 of the 7 examined clones contained the $\sim 1500$ bp Fab $\left(\mathrm{V}_{\mathrm{H}} \mathrm{C}_{\mathrm{H}} 1\right.$ $\mathrm{V}_{\mathrm{L}} \mathrm{C}_{\mathrm{L}}$ ) fragment.

\section{Library construction}

The Fab library was constructed in two steps. In the first step, the $\kappa$ light chain fragments were digested by restric- tion enzymes $S a c \mathrm{I} / X b a \mathrm{I}$, gel-purified, and ligated to the phagemid vector $\mathrm{pComb3H-SS}$ that had been cut with the same enzymes. The schematic structure of pComb3H-SS is shown in Figure 3 [17]. Following precipitation and washing, the $\kappa$ ligation mixture was electroporated into $E$. Coli XL1-Blue to form the $\kappa$ light chain gene library. The total number of transformants was about $4.5 \times 10^{7}$. Examination of plasmids from nine randomly selected XL1-Blue clones revealed that all nine clones contained the expected 680 bp insert (Figure 4A). Thus the size of the $\kappa$ light chain library was $\sim 4.5 \times 10^{7}$. In the second step, the heavy chain $\mathrm{Fd}$ fragments were digested with $\mathrm{XhoI} / \mathrm{SpeI}$ and cloned into the vector already containing the $\kappa$ light chain repertoires. After transforming XL1-Blue, the Fab library was formed and the total number of transformants reached $3.5 \times 10^{7}$. Examination of plasmids from nine randomly selected XL1-Blue clones showed that seven of these contained the expected insert of 680 bp (Figure 4B). By digestion with $S a c$ I / SpeI, five of these seven plasmids released the expected insert of $\sim 1500 \mathrm{bp}$ (Figure 4C). Thus we estimated that the actual diversity of our Fab library was $\sim 1.94 \times 10^{7}$ $\left(3.5 \times 10^{7} \times 78 \% \times 71 \%\right)$.

\section{Phage Enrichment}

After five rounds of biopanning, the percentage of phage yield increased from $7.50 \times 10^{-5}$ to $1.80 \times 10^{-2}$, representing an enrichment of about 200 fold (Table 2) as a result of successive removal of low affinity and nonspecifically bound phages.

Selection of soluble Fab antibodies against B lymphoma cell lines by ELISA

After the final round of biopanning, 65 clones were tested by ELISA against Raji cells, Daudi cells and BSA for the production of soluble Fab fragments. Finally, eight clones that showed high reactivity against Raji or Daudi cells with low background reaction against BSA were selected as positive clones. Among the eight positive clones, six showed high reactivity to both Raji and Daudi cells, and two only to Raji cells but not to Daudi cells. The eight positive Fabs were also tested against the pancreatic cancer cell line Patu 8988 and lung cancer cell line A549, and none of these clones demonstrated significant cross-reactivity with these two cell lines (Table 3).

\section{Sequence analysis of specific positive clones}

From the eight positive clones, FabC06, FabC21, FabC43 and FabC59 were selected for sequence analysis. The results of a homology search showed that the $\mathrm{V}_{\mathrm{H}}$ chains of FabC06, FabC21, FabC43 and FabC59 share 92\%, 89\%, $88 \%$ and $92 \%$ homology in amino acid sequences with the closest mouse Ig heavy chain variable region germline 
A $\quad \mathrm{VH}_{\mathrm{H}}$ amino acid sequences

Clones

P01807

FabC06

FabC21

FabC43

FabC59

Clones

P01807

FabC06

FabC21

FabC43

FabC59

Clones

P01807

FabC06

FabC21

FabC43

FabC59

Clones

P01807

FabC06

FabC21

FabC43

FabC59

EVKL L E S G G G L V Q

FR1

E K L LES G G G L V Q P G G S L K L S C A A S GF D F S

$--\mathrm{Q}----------------------$

$--\mathrm{Q}-------------------$

$--\mathrm{Q}--------------------$

$--Q---------------------$

FR2

W V R Q A P GK G L E W I G

CDR2

$-----------$

$-----------$

$----------$

$-----------$

\section{FR3}

K F I I S R D N A K N T L Y L Q M S K V R S E D T A L Y Y C A R $--------------------------$ $--------\mathrm{S}-------\mathrm{F}--\mathrm{A}------$ - - - - - - - - - - - - - - - - - - - - - $----------\mathrm{H}-------------$

\section{CDR3}

LHYYGYAAY**

$\mathrm{RD}-\mathrm{AQF}-\mathrm{Y} * * *$

GITFA-******

QTGTW-YTLDY

HSLLRLP $--* *$
E I N P D S S T I N Y T P S L K D

$----\mathrm{N} \mathrm{N}_{-}------$

- - - - - - - - - - - -

$-----------1$

$------------1$
CDR1

R Y W M S

$----$

$----$

$---1$

$---$

$B \quad V_{L}$ amino acid sequences

\section{Clones FR1}

AAA38888 VLTQSPA T L S V T P G D S V S L S C

FabC06 RARVDA - A - - - - - N - - - -

FabC21 RARVDA $-\mathrm{A}----\mathrm{L}-------$

FabC43 RARVDA $-\mathrm{A}-----\mathrm{N}-----$

FabC59 RARVDA $-\mathrm{A}-----------$

Clones

AAA38888

FabC06

FabC21

FabC43

FabC59

Clones

AAA38888

FabC06

FabC21

FabC43

FabC59

Clones

AAA38888

FabC06

FabC21

FabC43

FabC59

\section{FR2}

W F Q Q K S H E S P R L L I K

$-\mathrm{Y}-----\mathrm{K}------$

$-\mathrm{Y}-----------$

$-\mathrm{Y}-\mathrm{L}---------$

$-\mathrm{Y}----------$

FR3

\section{CDR3}

Q Q S N S W P F T *

$------\mathrm{Y}-*$

$-----P$ F T

$-\mathrm{H}-\mathrm{W}$ E I $-\mathrm{W}-*$

$------\mathrm{L}-*$
G I P S R F S G S G S G T D F T L S I N S V E T E D F G M Y F C

$-------------\mathrm{T}--\mathrm{N}------V---$

$---------------------$

$-----------N-$ H P - E $--\mathrm{T}_{\mathrm{A} \mathrm{T}}-\mathrm{Y}-$

$------------------------$
FR4

F G G G T K L E I K R A D A A

$-------\mathrm{R}----$

$--\mathrm{S}---------$

$-----------$

$--\mathrm{A}----\mathrm{L}-----$

Figure 5 Alignment of the deduced amino acid sequences of the $\mathrm{V}_{\mathrm{H}}$ and $\mathrm{V}_{\mathrm{L}}$ domains of the mouse Fab Clone 06 (FabC06), Fab Clone 21 (FabC21), Fab Clone 43 (FabC43) and Fab Clone 59 (FabC59) with the closest germline sequence. (A) $\mathrm{V}_{\mathrm{H}}$ sequences were compared with the closest mouse Ig heavy chain variable region germline sequence (Accession No. P01807). (B) $\mathrm{V}_{\mathrm{L}}$ sequences were compared with the closest mouse Ig kappa variable region germline sequence (Accession No. AAA38888.1). A dash (-) denotes identity to the uppermost sequence; * indicates no amino acid at this position. CDRs and FRs represent the sequences for complementary determining regions and framework regions respectively. 


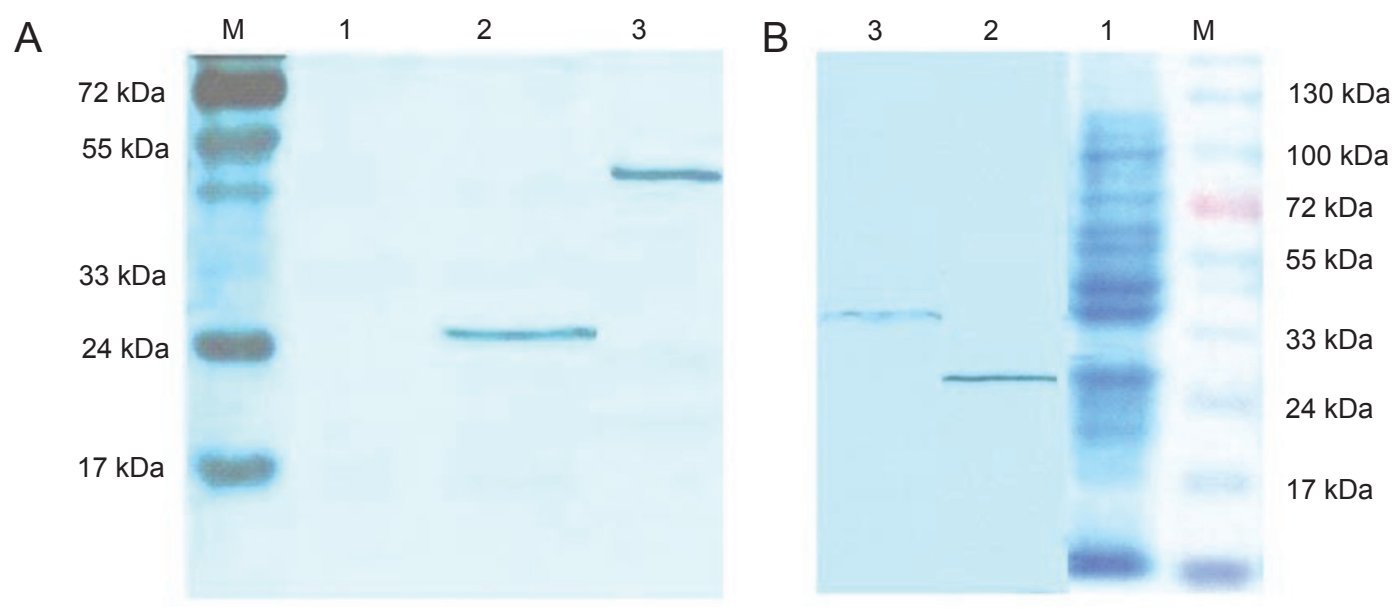

Figure 6 Western blot analysis. (A) Profile of the Fab antibody from FabC06. Lane M: Protein marker; Lane 1: Negative control of pComb3HSS transformed bacterial cell lysates; Lane 2: Fab under reducing conditions; Lane 3: Fab under non-reducing conditions. (B) The specificity of Fab antibodies toward membrane antigens of the B lymphoma cells analyzed by western blot. Lane M: Protein marker; Lane 1: Protein profile of Raji cells analyzed by reducing SDS-PAGE; Lane 2: Western blot of Raji cell proteins with the Fab antibody from FabC21; Lane 3: Western blot of Raji cell proteins with the Fab antibody from FabC06.
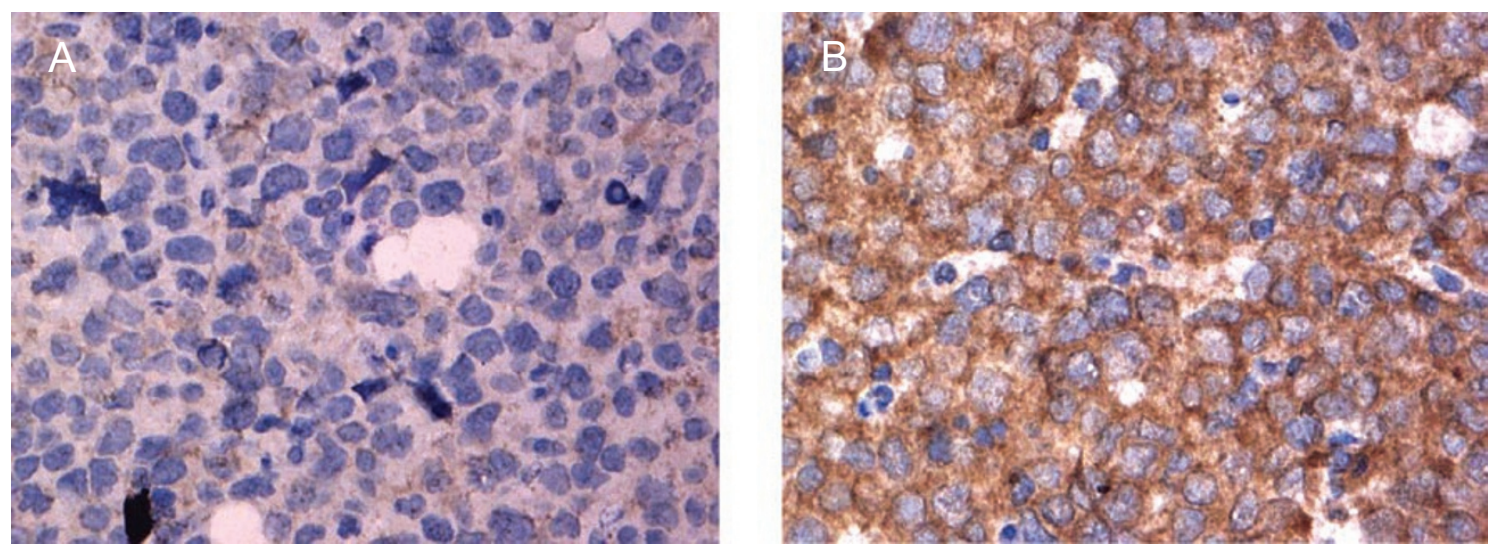

Figure 7 Immunohistochemical analysis of B lymphoma section with a soluble Fab antibody. (A) Negative control (original magnification: $\times 400$ ); (B) The positive reaction of FabC06 was found on the membranes of B lymphoma cells (original magnification: $\times 400$ ).

sequence (Accession No. P01807) respectively. $V_{L}$ chains of FabC06, FabC21, FabC43 and FabC59 share 89\%, 94\%, $81 \%$ and $94 \%$ homology in amino acid sequences with the closest mouse Ig kappa variable region germline sequence (Accession No. AAA38888.1) respectively. None of the $\mathrm{V}_{\mathrm{H}}$ and $\mathrm{V}_{\mathrm{L}}$ sequences of the four clones were identical to each other. The three complementary determining regions (CDRs) and four framework regions (FRs) were marked for $V_{H}$ chains and $V_{L}$ chains in Figure 5. As for the CDRs, CDR3 of the heavy variable domain showed the most frequent nucleotide and amino acid substitutions. On the other hand, only minor substitutions occurred in FRs. These results suggest that CDR3 of the heavy variable domain is associated with Fab function, which is the capability to recognize different antigens on the membrane of $\mathrm{B}$ lymphoma cells.

\section{Analysis of Fab antibodies by SDS-PAGE and western blot}

To determine the molecular weight of Fab antibodies, soluble Fab antibodies from FabC06 were resolved by SDSPAGE under both reducing and non-reducing conditions, and were subsequently detected by western blot analysis. As shown in Figure 6A, under non-reducing conditions, the 
soluble Fab appeared as a heterodimer (about $50 \mathrm{kDa}$ ) consisting of $\mathrm{Fd}$ and the light chain. Under reducing conditions, both $\mathrm{Fd}$ and the light chain were detected in monomeric form (about $25 \mathrm{kDa}$ ). These results indicated that the light chain and the heavy chain Fd were correctly linked by disulfide-bonding. The soluble Fab antibodies from FabC06 reacted mainly with a band at approximately $33 \mathrm{kDa}$ on the membrane of Raji cells, while soluble Fab antibodies from $\mathrm{FabC} 21$ reacted with a band at approximately $25 \mathrm{kDa}$ (Figure 6B). These results showed that the two soluble Fab antibodies from $\mathrm{FabC} 06$ and $\mathrm{FabC} 21$ recognized different membrane antigens of B lymphoma cells. Similar results were obtained for Daudi cells (data not shown).

\section{Analysis of Fab reactivity by immunohistochemistry}

Under the described condition for immunohistochemistry analysis, strong immunoreactivty for the Fabs was observed on the membrane of B lymphoma cells (Figure 7). However, no immunoreactivity was observed in the wider parts of the cytoplasm or in the nucleoli of B lymphoma cells.

\section{Discussion}

The emergence of phage display technique presents a new approach in tumor immunotherapy. Several phage antibody libraries against tumors have been constructed and screened, such as those against melanoma, osteosarcoma and prostate cancer $[7,20,21]$; and some of these phage antibodies showed high affinity and specificity for tumor antigens. As for lymphoma, so far only two studies have reported the generation of specific phage display library. Bruenke et al. [22] generated bispecific single-chain Fv antibody (bsscFv) from hybridomas $3 \mathrm{G} 8$ and F3.3 using the phage display library, which could mediate specific lysis of malignant human B-lymphoid cell lines. In another report, Dong et al. [23] isolated two Fab clones against the viral capsid antigen (VCA) of Epstein-Barr virus (EBV) from a patient with marginal zone $\mathrm{B}$ cell lymphoma.

In this study, we extracted total RNA from spleen cells of BALB/c mice immunized with Raji and Daudi cells. cDNAs coding for the $\kappa$ light chains and heavy chain $\mathrm{Fd}$ fragments were amplified by RT-PCR, and the amplified products were then used to construct the phage display library. It has been shown that when the diversity of an antibody phage display library reaches $1 \times 10^{7}$ individual clones, it will contain $99 \%$ of all antibody molecules. The size of antibody libraries that have been constructed so far generally ranges between $10^{6}$ and $10^{8}$ clones $[24,25]$. The size of our library for B cell lymphoma is $\sim 1.94 \times 10^{7}$ , which is of medium size and should satisfy the needs for covering the diversity of antibodies [26]. Following several rounds of biopanning, phages producing specific antibody molecules could be enriched [27]. Our study showed that the phage Fab libraries were enriched by about 200 fold after five rounds of biopanning with Raji cells and Daudi cells. ELISA analysis showed that the phage displaying Fab had significant binding activity with antigens associated with B cell lymphoma. Finally, eight clones that showed high reactivity with Raji cells or Daudi cells were selected as positive clones. To verify the origin of the Fab antibody genes and to estimate the extent of sequence diversity, amino acid sequences from four positive clones were aligned and compared with their closest murine germline $V$ regions. $V_{H}$ and $V_{L}$ domains were found to share $88-92 \%$ and $89-94 \%$ homology with the closest murine germline $\mathrm{V}$ regions respectively. As the $\mathrm{V}_{\mathrm{H}}$ domain of an antibody is known to play a major role in antigen recognition and binding [28], and also as somatic mutations in $\mathrm{V}$ genes during $\mathrm{B}$ cell maturation are indispensable for rearranged antibodies to obtain high specificity and affinity [29], we further analyzed the $\mathrm{V}_{\mathrm{H}}$ sequences of the four positive clones. We found that frequent amino acid substitutions occurred in CDR3, but only minor substitutions occurred in FRs. Western blot analysis showed that different Fab antibodies reacted with different membrane proteins of $\mathrm{B}$ lymphoma cells (Figure 6). It is likely that differences in CDR3 sequences may underlie the ability of respective Fab antibodies to recognize different antigens.

Today, mAbs are generated either by the hybridoma technology or from antibody libraries [30]. For practical reasons, the hybridoma technology is always confined to mice, thus making it difficult to use the resulting monoclonal antibodies for therapeutic purposes. Such antibodies may trigger human anti-mouse antibody (HAMA) response, especially when multiple infusions are required to obtain therapeutic efficacy $[2,3]$. Antibody libraries allow the generation of mAbs from virtually any species whose immunoglobulin genes are known. In this way, potential problems associated with the hybridoma approach, such as HAMA, low fusion, instability and low antibody production, will be resolved. Antibody libraries have been used to exploit large naive or synthetic antibody repertoires, or combinations of both, for the generation of human mAbs $[31,32]$. In contrast to antibodies derived from large naive or synthetic repertories, however, antibodies from immunized animals are subjected to in vivo selection and thus are more likely to selectively recognize a given antigen without cross-reactivity to another antigen [7]. In this study, we chose spleen cells of mice immunized with B lymphoma cells to construct phage display library. Though this method can reduce the diversity of antibodies against the target antigen, it should increase the proportion of specific antibodies and enhance the possibility of obtaining high affinity 
antibodies in a relatively small-size library. However, we can not directly construct human antibody phage library from lymphoma patients, because patients with lymphoma always have defects in humoral immunity, rendering it difficult to construct an effective phage antibody library against lymphoma.

In order to ensure the diversity of our phage antibody library, we took the following measures: firstly, total RNA was extracted from spleen of immunized mice to reduce the chance of RNA degradation; secondly, among the primers of Scripps Research Institute, twenty primers were designed, which included nine pairs of primers for subset IgG1, IgG2a and IgG3 respectively and seven pairs of primers for murine kappa light chain; thirdly, since the $\mathrm{V}_{\mathrm{H}}$ domain of an antibody is known to play a major role in antigen recognition and binding [28], the light chain was cloned first, followed by the heavy chain; and finally, the library size was improved by increasing the electroporation efficiency and repeating electroporation.

Standard phage display techniques depend upon panning of Fab-producing phages against purified antigen immobilized on substrates. These conditions may not be applicable if the antigen is not available in purified form or if it changes conformation under immobilization conditions. There are other cases, such as autoimmunity, in which the antigen itself may be unknown. In all of these cases, it would be difficult or impossible to apply traditional panning techniques for the identification of reactive antibodies of interest. This situation was encountered in our study, because the screening of our phage antibody library needed to be performed against membrane-bound antigens on $\mathrm{B}$ lymphoma cells. Some authors have successfully applied the phage display technology in screening, isolation and identification of tumor-associated cell surface antigens, such as those of melanomas, prostate cancer and colorectal carcinomas [7, 33, 34]. Various differentiation antigens are exposed on the membrane of B cell lymphoma, such as CD20, CD19 and CD22. It is worth noting that $95 \%$ of B lymphoma patients have high levels of CD20 on the membranes of tumor cells [35], while there is no exposure on cells of the normal tissue and multipotent stem cells. Thus, in our present study, we developed a method of cell-based panning to screen the Fab phage library, using six well plates coated by Raji and Daudi cells. After five rounds of panning, the enrichment was about 200 fold and ELISA assays identified eight positive clones.

In conclusion, we have successfully constructed a phage display antibody library for B cell lymphoma with fairly good sensitivity and specificity. These immunized Fab antibodies would lay a valuable experimental foundation for further study of B cell lymphoma and may also contribute to the improvement in the therapy of this disease.

\section{Acknowledgments}

We thank our colleagues Yujie Cui for technical assistance in electroporation and Jundong Zhou for immunizing the mice. This work was supported by grants from the National Natural Science Foundation of China (No. 30400111) and the Natural Science Foundation of Jiangsu Province (No.BK2004041).

\section{References}

1 Kohler G, Milstein C. Continuous cultures of fused cells secreting antibody of predefined specificity. Nature 1975; 256:495- 497.

2 Schroff RW, Foon KA, Beatty SM, Oldham RK, Morgan AC Jr. Human anti-murine immunoglobulin responses in patients receiving monoclonal antibody therapy. Cancer Res 1985; 45:879-885.

3 Shawler DL, Bartholomew RM, Smith LM, Dillman RO. Human immune response to multiple injections of murine monoclonal IgG. J Immunol 1985; 135:1530-1535.

4 Reff ME, Hariharan K, Braslawsky G. Future of monoclonal antibodies in the treatment of hematologic malignancies. Cancer Control 2002; 9:152-166.

5 Jones ML, Barnard RT. Chimerization of multiple antibody classes using splice overlap extension PCR. Biotechniques 2005; 38:181-182.

6 Dall'Acqua WF, Damschroder MM, Zhang J, et al. Antibody humanization by framework shuffling. Methods 2005; 36:4360.

7 Popkov M, Rader C, Barbas III CF. Isolation of human prostate caner cell reactive antibodies using phage display technology. J Immunol methods 2004; 291:137-151.

8 Maloney DG. Immunotherapy for non-Hodgkin's lymphoma: monoclonal antibodies and vaccines. J Clin Oncol 2005; 23:64216428 .

9 Tobinai K. Antibody therapy for malignant lymphoma. Intern Med 2007; 46:99-100.

10 Marcus R, Hagenbeek A. The therapeutic use of rituximab in non-Hodgkin's lymphoma. Eur J Haematol Suppl 2007; 67:514.

11 Batra SK, Jain M, Wittel UA, Chauhan SC, Colcher D. Pharmacokinetics and biodistribution of genetically engineered antibodies. Curr Opin Biotechnol 2002; 13:603-608.

12 Krauss J. Recombinant antibodies for the diagnosis and treatment of cancer. Mol Biotechnol 2003; 25:1-17.

13 Itoh K. Development of diagnostically and therapeutically useful human antibody medicines by phage display system. Yakugaku Zasshi 2007; 127:43-53.

14 Larralde OG, Martinez R, Camacho F, et al. Identification of hepatitis A virus mimotopes by phage display, antigenicity and immunogenicity. J Virol Methods 2007; 140:49-58.

15 De Pascalis R, Gonzales NR, Padlan EA, et al. In vitro affinity maturation of a specificity-determining region-grafted humanized anticarcinoma antibody: isolation and characterization of minimally immunogenic high-affinity variants. Clinical Cancer Research 2003; 9:5521-5531.

16 Wei Chan S, Ong GI and Nathan S. Neutralizing chimeric mousehuman antibodies against Burkholderia pseudomallei protease: 
expression, purification and characterization. Biochem Mol Biol 2004; 37:556-564.

17 Andris-Widhopf J, Rader C, Steinberger P, Fuller R, Barbas CF $3^{\text {rd }}$. Methods for the generation of chicken monoclonal antibody fragments by phage display. J Immunol Methods 2000; 242:159181.

18 Orum H, Andersen PS, Oster A, et al. Efficient method for constructing comprehensive murine Fab antibody libraries displayed on phage. Nucleic Acids Res 1993; 21:4491-4498.

19 de Alboran IM, Martinez-alonso C, Barbas CF $3^{\text {rd }}$, Burton DR, Ditzel HJ. Human monoclonal Fab fragments specific for viral antigens from combinatorial IgA libraries. Immunotechnology $1995 ; \mathbf{1}: 21-28$.

20 Li J, Pereira S, Van Belle P, et al. Isolation of the melanoma-associated antigen $\mathrm{p} 23$ using antibody phage display. J Immunol 2001; 166:432-438.

21 Dantas-Barbosa C, Brigido MM, Maranhao AQ. Construction of a human Fab phage display library from antibody repertoires of osteosarcoma patients. Genet Mol Res 2005; 4:126-140.

22 Bruenke J, Fischer B, Barbin K, et al. A recombinant bispecific single-chain Fv antibody against HLA class II and FcgrmmaRIII (CD16) triggers effective lysis of lymphoma cells. Br J Haematol 2004; 125:167-179.

23 Dong L, Masaki Y, Takegami T, et al. Cloning and expression of two human recombinant monoclonal Fab fragments specific to EBV capsid antigen. Int Immunol 2007; 19:331-336.

24 Wu BP, Xiao B, Wan TM, Zhang YL, Zhang ZS, Zhou DY. Construction and selection of the natural immune Fab antibody phage display library from patients with colorectal cancer. World J Gastroenterol 2001; 7:811-815.

25 Nathan S, Rader C, Barbas CF. Neutralization of Burkholderia pseudomallei protease by Fabs generated through phage display.
Biosci Biotechnol Biochem 2005; 69:2302-2311.

26 Smith JW, Ruoslahti E. Harvesting molecular diversity-biology's new commodity. Biotechnol Genet Eng Rev 1997; 14:51-65.

27 Watters JM, Telleman P, Junghans RP. An optimized method for cell-based phage display panning. Immunotechnology 1997;3: 21-29.

28 Pereira S, Maruyama H, Siegel D, et al. A model system for detection and isolation of a tumor cell surface antigen using antibody phage display. J Immunol Methods 1997; 203:11-24.

29 Manser T, Tumas-Brundage KM, Casson LP, et al. The roles of antibody variable region hypermutation and selection in the development of the memory B-cell compartment. Immunol Rev 1998; 162:183-196.

30 Rader C. Antibody libraries in drug and target discovery. Drug Discov Today 2001; 6:36-43.

31 Hoogenboom HR, Chames P. Natural and designer binding sites made by phage display technology. Immunol Today 2000; 21:371-378.

32 Desogus A, Burioni R, Ingianni A, Bugli F, Pompei R, Fadda G. Production and characterization of a human recombinant monoclonal Fab fragment specific for influenza A viruses. Clin Diagn Lab Immunol 2003; 10:680-685.

33 Kupsch JM, Tidman NH, Kang NV, et al. Isolation of human tumor-specific antibodies by selection of an antibody phage library on melanoma cells. Clin Caner Res 1999; 5:925-931.

34 Mutuberria R, Stijn S, Huijbers A, et al. Isolation of human antibodies to tumor-associated endothelial cell markers by in vitro human endothelial cell selection with phage display libraries. J Immunol Methods 2004; 287:31-47.

35 Press OW, Farr AG, Borroz KI, Anderson SK, Martin PJ. Endocytosis and degradation of monoclonal antibodies targeting human B-cell malignancies. Cancer Res 1989; 49:4906-4912. 